\title{
Oxygen vacancies effect on phase separation in $\mathrm{Pr}_{0.5} \mathrm{Ca}_{0.2} \mathrm{Sr}_{0.3} \mathrm{MnO}_{3-\delta}$
}

\author{
D. Niebieskikwiat, ${ }^{\text {a) }}$ A. Caneiro, and R. D. Sánchez \\ Comisión Nacional de Energía Atómica-Centro Atómico Bariloche and Instituto Balseiro, \\ 8400 Bariloche, Argentina
}

(Presented on 14 November 2002)

\begin{abstract}
We study the effect of the introduction of a controlled amount of oxygen vacancies on the magnetic and transport properties of the phase separated manganite $\operatorname{Pr}_{0.5} \mathrm{Ca}_{0.2} \mathrm{Sr}_{0.3} \mathrm{MnO}_{3-\delta}$, for $\delta=0$ and 0.01 . This compound presents a paramagnetic to ferromagnetic (FM) transition below $T_{C} \sim 240 \mathrm{~K}$, and then to a charge-ordered $(\mathrm{CO})$ and antiferromagnetic $(\mathrm{AFM})$ phase at $T_{\mathrm{CO}} \sim 175 \mathrm{~K}$. However, below $T_{\mathrm{CO}}$ an appreciable FM component $(\sim 20 \%)$ still survives, related to a FM volume immersed within the $\mathrm{CO} / \mathrm{AFM}$ matrix. At low temperatures, in the mainly $\mathrm{CO}$ phase, the introduction of oxygen vacancies enhances the FM ordering, with the consequent decrease of resistivity and magnetoresistance (MR). On the contrary, in the FM phase (in the range $T_{\mathrm{CO}}<T<T_{C}$ ) the magnetization is reduced and the resistivity and MR increase. These results are discussed in the frame of percolative transport in metal-insulator mixtures, and frustrated double exchange interaction due to the interruption of some $\mathrm{Mn}-\mathrm{O}-\mathrm{Mn}$ chains. (C) 2003 American Institute of Physics. [DOI: 10.1063/1.1540182]
\end{abstract}

\section{INTRODUCTION}

The study of magnetic and transport properties of phase separated manganites of the type $A_{1-x}^{3+} A_{x}^{\prime 2+} \mathrm{MnO}_{3}$ has become, in the recent past, a very important topic in the area of transition metal oxides. ${ }^{1-10}$ The most attractive properties of the phase separation (PS) phenomenon are the percolativetype insulator-to-metal (IM) transitions and the high "colossal magnetoresistance" generated by the growth of the ferromagnetic (FM) clusters when a magnetic field $(H)$ is applied. ${ }^{3,4}$ These FM domains have been found to coexist either with paramagnetic (PM) or charge-ordered antiferromagnetic (CO/AFM) phases, depending on the doping level $x$ and the temperature $(T)$ range. ${ }^{5}$ Although several theoretical attempts have been made to understand the origin of the PS, ${ }^{1,6-8}$ there are no conclusive experiments about this point. In this sense, there are some claims that the oxygen stoichiometry could play an important role. ${ }^{8-10}$ In low-doped $\mathrm{La}_{1-x} \mathrm{Ca}_{x} \mathrm{MnO}_{3}$, an increase of the oxygen content produces an IM transition due to the percolation of the FM metallic phase. ${ }^{9}$ On the other hand, in $\mathrm{La}_{0.5} \mathrm{Ca}_{0.5} \mathrm{MnO}_{3}$, the introduction of oxygen vacancies just induces a decrease of the resistivity at low $T .^{10}$

In the present work we study the influence of a small amount of oxygen vacancies on the electric and magnetic properties of $\operatorname{Pr}_{0.5} \mathrm{Ca}_{0.2} \mathrm{Sr}_{0.3} \mathrm{MnO}_{3-\delta}$, for $\delta=0$ and 0.01 .

\section{EXPERIMENT}

Magnetization $(M)$ data were recorded in a commercial superconducting quantum interference device magnetometer in the 5-300 $\mathrm{K}$ temperature range with applied magnetic fields up to $50 \mathrm{kOe}$. Four-probe resistivity $(\rho)$ was measured between 5 and $300 \mathrm{~K}$ for $H=0$ and $90 \mathrm{kOe}$. The sample

\footnotetext{
a) Author to whom correspondence should be addressed; electronic mail: niebied@cab.cnea.gov.ar
}

preparation and characterization was described elsewhere. ${ }^{3}$ For the oxygen control, the equilibrium oxygen partial pressure $\left[p\left(\mathrm{O}_{2}\right)\right]$ data as a function of $\delta$ were obtained at $T$ $=1000{ }^{\circ} \mathrm{C}$ through thermogravimetric measurements. ${ }^{11}$ The as-made sample was found to be stoichiometric $(\delta=0)$, while the $\delta=0.01$ sample was prepared by annealing the $\delta$ $=0$ one at $T=1000^{\circ} \mathrm{C}$ and $p\left(\mathrm{O}_{2}\right)=1.03 \times 10^{-5}$ atm during $24 \mathrm{~h}$, and later quenching at liquid nitrogen temperature.

\section{RESULTS AND DISCUSSION}

In the inset of Fig. 1 we show $M$ vs $H$ curves of the two samples studied at two selected temperatures. From the back extrapolation of these curves to zero magnetic field we obtained the spontaneous magnetization $M_{0}$ as a function of temperature, as shown in the main panel of the same figure. In phase separated manganites, $M_{0}$ is directly proportional to the FM phase fraction $(X),{ }^{6}$ thus it allows a direct quantitative comparison of the two samples studied. As observed in the $M_{0}(T)$ data, at $T_{C} \sim 240 \mathrm{~K}$ there is a PM to FM transition, and lowering $T$ below $T_{\mathrm{CO}} \sim 175 \mathrm{~K}$ there is a FM to $\mathrm{CO} / \mathrm{AFM}$ transition. However, even at $T=10 \mathrm{~K}$ the magnetization loops in the inset of Fig. 1 continue showing a FMlike behavior, with a reduced $M_{0} \approx 0.78 \mu_{B}$ and $0.89 \mu_{B}$ for $\delta=0$ and 0.01 , respectively. This behavior has been interpreted as the signature of the coexistence of the FM and $\mathrm{CO} / \mathrm{AFM}$ phases. ${ }^{6}$ When compared to the saturation magnetization $M_{s} \approx 3.5 \mu_{B}$, the values of $M_{0}$ indicate that the FM phase fraction at low $T$ is increased from $X \approx 22 \%$ for $\delta$ $=0$ to $\approx 25 \%$ for $\delta=0.01$.

For temperatures around $200 \mathrm{~K}$, it has been shown that the $\delta=0$ compound is almost totally $\mathrm{FM}^{3}$ The $M_{0}(T)$ curves of Fig. 1 show that, in the FM phase, the introduction of oxygen vacancies causes a reduction of the magnetization, as opposed to the low temperature behavior. This result is fully compatible with the resistivity data, which are shown as a function of $T$ in Fig. 2. As can be observed, for $T>T_{\mathrm{CO}}$ the 


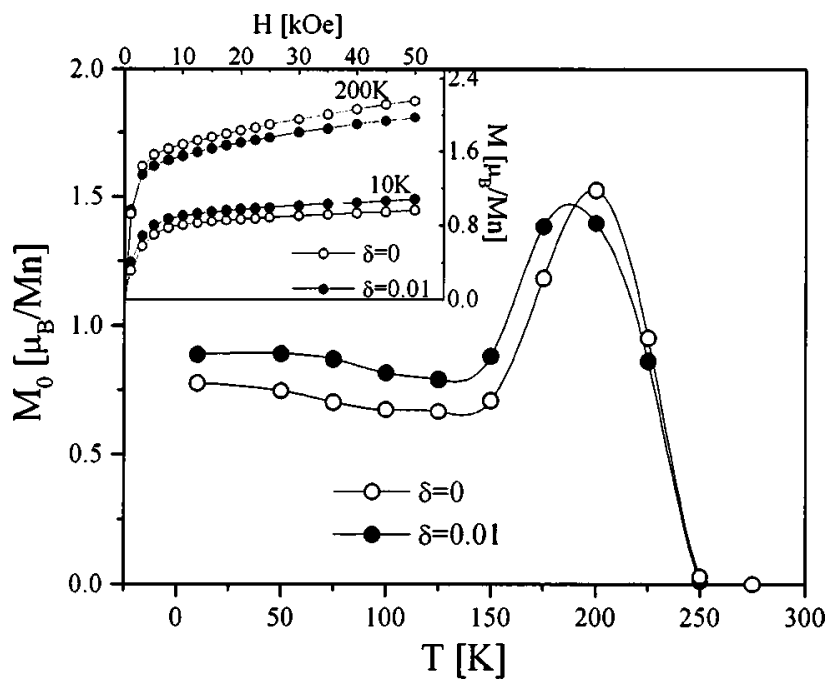

FIG. 1. Spontaneous magnetization $\left(M_{0}\right)$ as a function of temperature for the $\operatorname{Pr}_{0.5} \mathrm{Ca}_{0.2} \mathrm{Sr}_{0.3} \mathrm{MnO}_{3-\delta}$ compound. (Inset) Magnetization vs field curves at two selected temperatures.

$\rho$ for $\delta=0.01$ is higher than the $\rho$ of the stoichiometric sample. The reduction of the ability for the charge transport can be interpreted as a partial suppression of the double exchange (DE) interaction. ${ }^{12}$ Due to the absence of some oxygen ions, some $\mathrm{Mn}-\mathrm{O}-\mathrm{Mn}$ paths for the conducting electrons are interrupted. The interruption of these chains induces an increase of resistivity, and at the same time the effective FM (DE) interaction between neighboring Mn sites is frustrated, thus reducing the magnetization.

Below $T_{\mathrm{CO}}$, the strong insulating state exhibited by the $\rho(T)$ curves in Fig. 2 is related to the transition to the mainly $\mathrm{CO} / \mathrm{AFM}$ phase at low $T$. However, the saturating resistivity below $T \sim 75 \mathrm{~K}$ in the $\delta=0$ sample has been shown to be induced by the remaining FM fraction $X \sim 22 \% .^{3}$ Notably, below the $\mathrm{CO}$ temperature, where the oxygen vacancies produce a magnetization increase, the $\rho$ for $\delta=0.01$ is reduced with respect to that for $\delta=0$, as opposed to the case $T$ $>T_{\mathrm{CO}}$. The increase of the FM phase fraction at low $T$ to $X \sim 25 \%$ in the $\delta=0.01$ sample favors the percolation of the

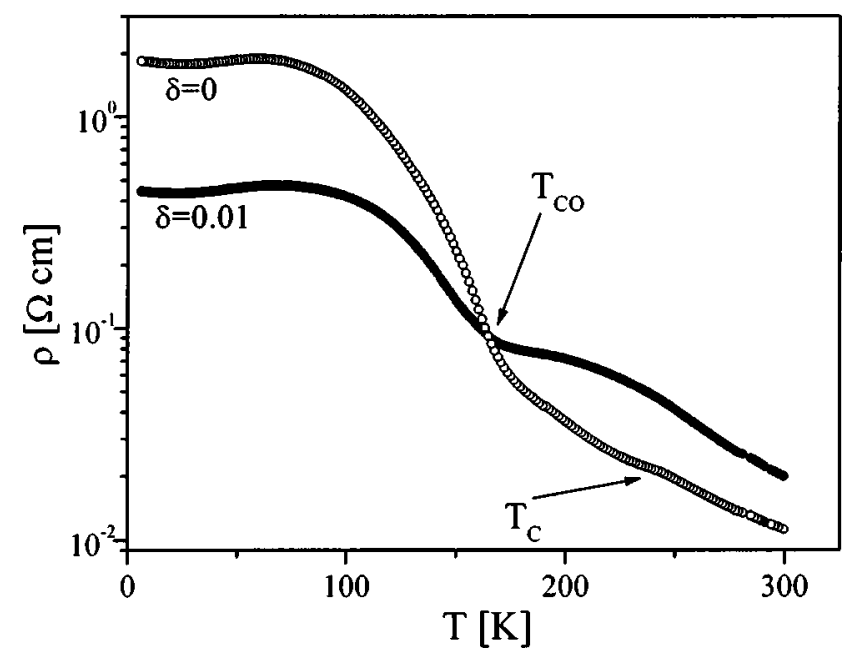

FIG. 2. Resistivity vs temperature curves for the two samples studied. metallic phase, with the consequent reduction of the resistivity. The enlargement of the FM volume indicates that when oxygen vacancies are introduced in the AFM host of the half-doped compound, these destabilize the AFM phase producing a FM polarization. A simple estimation can be made about the volume polarized by each oxygen vacancy. If we assume a homogeneous distribution of the oxygen vacancies throughout the sample, a fraction $X$ of them will lie within the FM volume, while the remaining fraction $(1-X)$ should be placed within the initially AFM volume. Then, when an amount $\delta$ of oxygen vacancies is introduced, the induced change of $M_{0}$ is given by

$$
\Delta M_{0}=\delta(1-X) m_{v A}+\delta X m_{v F},
$$

where $m_{v A}$ (or $m_{v F}$ ) is the magnetic moment induced by each oxygen vacancy located in the initially AFM (or FM) volume. As mentioned before, the vacancies located in a FM host reduce the magnetization, thus the second term of Eq. (1) gives a negative contribution to $\Delta M_{0}\left(m_{v F}<0\right)$. At $T$ $=200 \mathrm{~K}$, where $X \sim 1$, we have $\Delta M_{0}(200 \mathrm{~K})$ $\approx-0.125 \mu_{B}$, implying that $m_{v F} \sim \Delta M_{0}(200 \mathrm{~K}) / \delta$ $=-12.5 \mu_{B}$. If we assume that $m_{v F}$ is not strongly temperature dependent, we can use Eq. (1) to obtain the value of $m_{v A}$ at $T=10 \mathrm{~K}$ as

$$
m_{v A} \sim \frac{\Delta M_{0}}{\delta(1-X)}-\frac{X}{(1-X)} m_{v F} .
$$

In fact, since at low $T$ we have that $X \ll(1-X)$, the precise value of $m_{v F}$ is not very important for an estimation of $m_{v A}$. By evaluating Eq. (2), we find that each vacancy in the AFM host induces a polarization $m_{v A} \sim 17.6 \mu_{B}$. Assuming that the effect of these vacancies is a full polarization of the surrounding $\mathrm{Mn}$ sites $\left(3.5 \mu_{B}\right.$ for each one), this $m_{v A}$ would correspond to a FM polarization of $\sim 5$ neighboring Mn cations. Of course this is a rough estimation of the effect of the oxygen vacancies. However, this result clearly indicates that, at low $T$, the oxygen vacancies have a tendency to produce a local FM ordering in their vicinity. This local ordering implies the formation of small FM polarons, with a correlation length of $\sim 1 \mathrm{~nm}$, similar to those observed in the slightly doped $\mathrm{La}_{1-x} \mathrm{Ca}_{x} \mathrm{MnO}_{3} \cdot{ }^{13}$

In Fig. 3 we present the ratio $\rho(H=90 \mathrm{kOe}) / \rho(H$ $=0$ ), i.e., the magnetoresistance at $H=90 \mathrm{kOe}$, as a function of temperature. What can be observed is that in the FM phase $\left(T_{\mathrm{CO}}<T<T_{C}\right)$ the MR is improved when the oxygen vacancies are introduced, i.e. the ratio $\rho(90 \mathrm{kOe}) / \rho(0)$ is lowered, while the opposite response is observed in the mainly $\mathrm{CO} / \mathrm{AFM}$ phase. Once again, the crossover between the two regimes occurs at $T_{\mathrm{CO}}$, like in the $M_{0}(T)$ and $\rho(T)$ curves. This different behavior at both sides of the $\mathrm{CO}$ temperature is related to the different MR mechanisms of the FM and PS states. In the high- $T$ FM phase, the $\delta=0$ sample exhibits the usual MR of polycrystalline FM manganites. ${ }^{14}$ In addition, due to the partially frustrated DE interaction, in the oxygen deficient sample there are some Mn sites with reduced magnetization acting like scattering centers. When a magnetic field is applied, these Mn sites are polarized and this scattering disappears, thus the relative reduction of resistivity is more important than in the $\delta=0$ sample. 


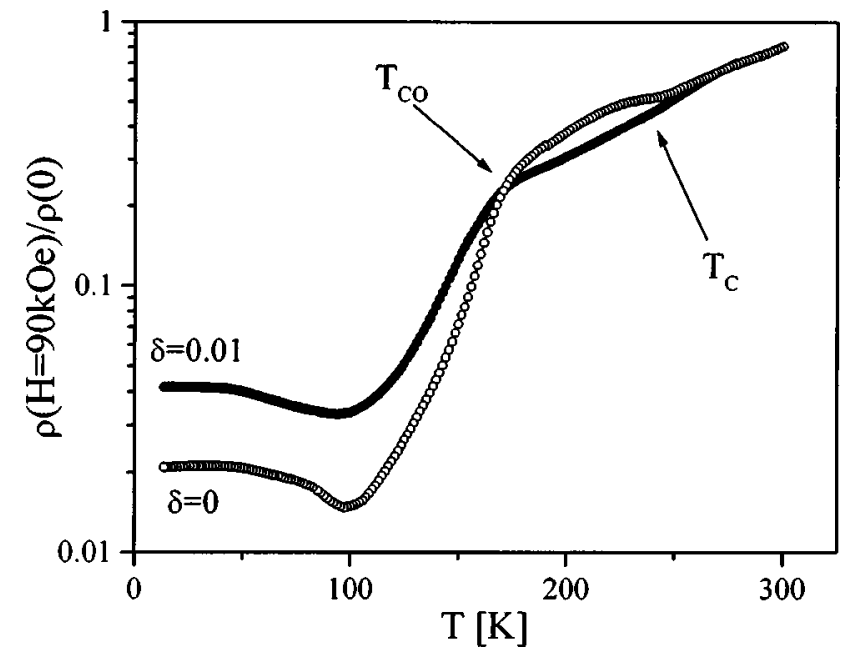

FIG. 3. Magnetoresistance $\rho(H) / \rho(0)$ at $H=90 \mathrm{kOe}$ as a function of temperature for the stoichiometric $(\delta=0)$ and oxygen-deficient ( $\delta=0.01)$ compounds.

In the PS low-temperature state the MR mechanism is different. In this case, the magnetic field induces the swelling of the FM clusters due to the gain of the Zeeman energy. The enlargement of the metallic volume improves the percolative paths for the current transport, then producing a huge decrease of $\rho$. The fact that the MR at low $T$ is more important for $\delta=0$ indicates that, in this sample, the increase of $X$ with $H$ is larger. There are two possibilities to explain this response. On one hand, it is possible that in the $\delta=0.01$ sample, due to the enlarged zero-field FM fraction, the further increase of FM volume, $\propto(1-X)$, becomes reduced, thus reducing the MR. However, this explanation is weakened by the fact that, in the $\operatorname{Pr}_{0.5} \mathrm{Ca}_{0.2} \mathrm{Sr}_{0.3} \mathrm{MnO}_{3}$ compound, fields much higher than $90 \mathrm{kOe}$ are necessary at low $T$ to reach the complete FM state $X=1 .{ }^{6}$ On the other hand, it is likely that the oxygen vacancies produce a pinning of the FM clusters, making their growth more difficult.

\section{SUMMARY}

In summary, we studied the electric and magnetic properties of the phase separated manganite $\mathrm{Pr}_{0.5} \mathrm{Ca}_{0.2} \mathrm{Sr}_{0.3} \mathrm{MnO}_{3-\delta}$, for $\delta=0$ and 0.01 . We show that the oxygen vacancies behave in a different way, depending on the predominant magnetic phase. In the mainly CO/AFM phase at low $T$, these vacancies exhibit a tendency to produce a local FM ordering in their vicinity, with the consequent reduction of the resistivity. On the contrary, in the FM phase above $T_{\mathrm{CO}}$ they act as scattering centers, where the double exchange interaction is locally suppressed due to the interruption of a $\mathrm{Mn}-\mathrm{O}-\mathrm{Mn}$ chain.

${ }^{1}$ E. Dagotto, T. Hotta, and A. Moreo, Phys. Rep. 344, 1 (2001), and references therein.

${ }^{2}$ M. Uehara, S. Mori, C. H. Chen, and S-W. Cheong, Nature (London) 399, 560 (1999).

${ }^{3}$ D. Niebieskikwiat, R. D. Sánchez, L. Morales, and B. Maiorov, Phys. Rev. B 66, 134422 (2002).

${ }^{4}$ M. Mayr, A. Moreo, J. A. Vergés, J. Arispe, A. Feiguin, and E. Dagotto, Phys. Rev. Lett. 86, 135 (2001).

${ }^{5}$ D. Niebieskikwiat, R. D. Sánchez, and A. Caneiro, J. Magn. Magn. Mater. 237, 241 (2001).

${ }^{6}$ D. Niebieskikwiat, R. D. Sánchez, A. Caneiro, and B. Alascio, Phys. Rev. B 63, 212402 (2001).

${ }^{7}$ A. Moreo, M. Mayr, A. Feiguin, S. Yunoki, and E. Dagotto, Phys. Rev. Lett. 84, 5568 (2000).

${ }^{8}$ E. L. Nagaev, Phys. Lett. A 218, 367 (1996).

${ }^{9}$ I. Kim, J. Dho, and S. Lee, Phys. Rev. B 62, 5674 (2000).

${ }^{10}$ Y. G. Zhao et al., Phys. Rev. B 65, 144406 (2002).

${ }^{11}$ F. Prado, R. D. Sánchez, A. Caneiro, M. T. Causa, and M. Tovar, J. Solid State Chem. 146, 418 (1999).

${ }^{12}$ C. Zener, Phys. Rev. 82, 403 (1951).

${ }^{13}$ M. Hennion, F. Moussa, J. Rodríguez-Carvajal, L. Pinsard, and A. Revcolevschi, Phys. Rev. B 56, R497 (1997); M. Hennion et al., Phys. Rev. Lett. 81, 1957 (1998).

${ }^{14}$ H. Y. Hwang, S-W. Cheong, N. P. Ong, and B. Batlogg, Phys. Rev. Lett. 77, 2041 (1996). 